Fecha de recepción: marzo 2020 Fecha de aceptación: abril 2020 Versión final: mayo 2020

\section{Abstracción, Transformación, e Inspiración. El arte como fuente de conocimiento y creatividad arquitectónica en el taller de diseño ${ }^{1}$}

Peggy McDonough ${ }^{(1)}$ y Julio Bermúdez ${ }^{(2)}$

\begin{abstract}
Resumen: Aunque se ha dicho mucho sobre la relación directa entre el arte y la arquitectura, poco se ha hecho sobre cómo las obras de arte históricamente significativas pueden ser utilizadas como fuentes de inspiración para la producción arquitectónica de hoy. Podemos citar varios análisis históricos y estéticos sobre tales obras de arte y su espíritu particular, pero ¿cómo podría una obra de arte de este tipo iluminar y extender el proceso arquitectónico, las teorías y las ideas actuales?

En otras palabras, (1) ¿cómo el pasado (in) (de) (re) forma el presente? y (2) ¿cómo las percepciones de otra disciplina (in) (de) (re) forman el proceso de ideación de nuestra disciplina? Teniendo en cuenta estos pensamientos, se desarrolló una pedagogía para permitir que estudiantes en el comienzo de la carrera de arquitectura se den cuenta de que (1) la arquitectura trata temas e ideas que trascienden lo puramente programático (es decir, 'funcional') y lo estereotípicamente formal; (2) las nuevas ideas arquitectónicas pueden provenir de fuentes no arquitectónicas del pasado; (3) las ideas se descubren y evolucionan a través de un proceso crítico por etapas, en oposición a un acto singular de explicación; y (4) el papel del arquitecto/diseñador es interpretar, en lugar de imitar, el entorno, la cultura, la tradición, etc.
\end{abstract}

Palabras claves: arte y arquitectura - pedagogía del diseño - proceso proyectual.

[Resúmenes en inglés y portugués en las páginas 46-47]

(1) Peggy McDonough: es arquitecta y presidenta de MHTN Architects Inc., una firma de arquitectura, planificación y diseño que ha prestado servicios en la región de Salt Lake City, Utah (EEUU) durante 95 años. Originaria de Utah, la arquitecta McDonough tiene 35 años de experiencia en arquitectura y diseño. Recibió su título profesional de la Universidad de Notre Dame (Indiana, EEUU) que incluyó un año de estudios de arquitectura y urbanismo en Roma, Italia. Como presidenta de MHTN, parte de su misión es expandir la forma en que los arquitectos sirven al contexto comunitario y natural. McDonough lidera un enfoque de diseño colaborativo que enfatiza a las personas, el lugar y la polinización entre múltiples experticias. Entre los muchos reconocimientos recibidos se destacan la Beca Anual Lloyd Warren / Premio de París en Arquitectura de 1989, y el premio de excelencia en diseño por el Centro de Visitantes de Antelope Island en el gran lago salado de Utah. De 1995 a 2015, se desempeñó como profesora auxiliar adjunta en la Facultad de 
Arquitectura + Planificación de la Universidad de Utah, y de 2001 a 2008 en la Comisión de Planificación de Salt Lake City.

(2) Julio Bermúdez dirige el programa de posgrado de Estudios Culturales y Espacio Sagrado en la Escuela de Arquitectura y Planificación de la Catholic University of America (Washington, DC, EEUU) desde 2010. Antes de este nombramiento, el Dr. Bermúdez fue profesor en la Universidad de Utah durante 17 años. Tiene una maestría en arquitectura y un $\mathrm{PhD}$ en educación de la Universidad de Minnesota. Ha enseñado diseño arquitectónico, teoría y representación por más de 30 años. Los intereses del Dr. Bermúdez se centran en la relación entre arquitectura, cultura y espiritualidad a través de la lente de la fenomenología y la neurociencia. Ha impartido conferencias, liderado simposios y publicado en estas áreas, incluyendo los libros "Transcending Architecture. Contemporary Views on Sacred Space" (CUA Press 2015) y "Architecture, Culture and Spirituality" (Routledge 2015). Su investigación actual se enfoca en experiencias arquitectónicas extraordinarias que permiten acceso a lo trascedente así como también en pedagogías que incorporan al desarrollo espiritual dentro de la educación profesional del arquitecto. El Dr. Bermúdez es presidente del Foro de Arquitectura, Cultura y Espiritualidad, una organización internacional que cofundó en 2007 y que cuenta con mas de 550 miembros de 52 países (http:// www.acsforum.org/9)

\section{Introducción}

\section{Arte y Arquitectura}

La fuerte relación entre el arte y la arquitectura ha sido ampliamente documentada. Colin Rowe y Robert Slutzky presentan un excelente argumento sobre este tema en su artículo en Inglés "Transparency: Literal \& Phenomenal" 2 . En este trabajo, sus autores demuestran el intenso diálogo entre la pintura cubista y la arquitectura moderna, enfocándose particularmente en los aspectos estéticos y técnicos de la transparencia y el fenómeno cubista de simultaneidad ${ }^{3}$. Esta investigación analiza cómo el arte puede informar a la arquitectura y, a su vez, cómo la arquitectura puede estimular la experiencia del arte. Se pueden encontrar influencias similares entre estilos artísticos y arquitectónicos a lo largo de la historia humana ${ }^{4}$.

Sin embargo, nuestro interés aquí no está en la reconstrucción de los paralelos históricos expuestos previamente entre el arte y la arquitectura. Lo que nos convoca es reconocer que, dada la fuerte relación simbiótica del arte y la arquitectura, uno podría usar conscientemente el arte (la pintura para los propósitos de esta discusión) como catalizador de nuevas ideas arquitectónicas. La utilización del arte como un medio para extender la investigación arquitectónica obviamente requeriría un salto metafórico o analógico entre los temas específicos que una obra de arte en particular confrontó en su momento con los 
que la arquitectura enfrenta en la actualidad. Es precisamente este salto (cuyo potencial trasciende lo puramente estético y pasa a lo técnico, filosófico, social o político) lo que es fascinante: la obra de arte se convierte en un instrumento para cambiar nuestra percepción habitual y así ver la arquitectura desde una nueva perspectiva. El arte, emocional y racional a la vez, emplea la reacción, el razonamiento, el rigor y la interpretación. La importancia del arte para iluminar, inspirar y avanzar la arquitectura ha sido respaldada por muchos arquitectos contemporáneos de renombre como Daniel Libeskind y Juhani Pallassma 5 .

El arte y la arquitectura, como actos creativos, también comparten la necesidad de aliviar la ansiedad psicológica de crear algo de la nada: la intimidación del lienzo en blanco. Las metodologías de artistas excepcionales demuestran superar este miedo y alcanzar un estado mental más abierto y original al inspirarse en áreas creativas diferentes pero relacionadas al trabajo a realizar. La coreógrafa estadounidense y creadora de obras iconoclastas en danza moderna, Twyla Tharp, se refiere a la paradoja creativa de la libertad y la restricción, y sus métodos para comenzar la ideación en una habitación blanca y vacía. El trabajo innovador de Tharp proviene de invitar la emoción y lo desconocido a un proceso creativo que es equilibrado por cuerpos y mentes precalentados por su propia constitución y la rutina $^{6}$. Su trabajo ha sido notablemente influenciado por el estudio de movimientos no relacionadas con la danza como el boxeo o la circulación peatonal urbana. Utilizando el arte como punto de partida, nuestra pedagogía estaba interesada en exponer a los estudiantes de arquitectura a la siguiente paradoja: crear es un hecho emocional que requiere una inspiración facilitada por una disciplina de trabajo y la continua interpretación analógica o metafórica-especialmente cuando dejamos de utilizar precedentes arquitectónicos para inspirar nuestra arquitectura.

\section{La Pintura como Modelo}

A diferencia de otras metodologías de diseño que involucran un ejemplar o modelo, como en el caso de la tipología arquitectónica, nuestro enfoque puede ofrecer formas mucho más originales y, por lo tanto, acceso a nuevas ideas arquitectónicas. En la presente discusión sobre el valor del precedente arquitectónico como disparador del proceso de diseño, se debe considerar la siempre presente tentación por parte del estudiante (y del profesional) de apropiarse o repetir “obras maestras," en un intento de dar valor a lo nuevo.

El uso del Tipo empuja la arquitectura a imitaciones de modelos lingüísticos en los que el significado finalmente se encuentra fuera de la obra misma. Las abstracciones a priori o los 'elementos' típicos (paredes, columnas, habitaciones, planos, etc.) tienen prioridad sobre las cosas reales. El diseño creativo se convierte en un proceso de correspondencia o cumplimiento con el Tipo en lugar de hacer o construir lo que es necesario ${ }^{7}$.

Aunque la pintura como obra cultural también podría prestarse a ser 'tipologeada,' esto se evita cuando uno la aplica o transfiere a otra disciplina. En esta situación, el usuario se ve 
obligado a profundizar más allá de lo superficial, desarrollar capacidades interpretativas, y determinar relevancias en relación a la búsqueda encomendada, en nuestro caso, la de la arquitectura.

No es el propósito de este trabajo sugerir que la pintura, o el arte en general, deba ser utilizada como modelo en lugar del modelo arquitectónico en el taller de diseño. Sin embargo, es instructivo considerar los posibles efectos positivos de recurrir a la pintura como modelo anterior o paralelo al uso de modelos arquitectónicos, sobre todo en un taller de diseño dirigido a principiantes de la arquitectura como el nuestro. En tal caso, el acto de interpretación se enfatizaría claramente sobre el acto de imitación.

Desde la perspectiva moderna/contemporánea que estamos tomando en este artículo, el origen auténtico del arte y la arquitectura no deviene de repetir versiones existentes de la realidad, generalmente heredadas del pasado y concretizadas por hábitos comunes de percepción o acción. Más bien, es la ver/hacer intencional o proactivo de algo aún nunca visto/hecho lo que logra revelarse como valioso. Esto requiere un proceso de valor agregado en el que la visión/lectura particular del artista es de importancia primordial. El verdadero arte requiere un filtrado intencional, un acto sesgado de (mayor o menor) sabia interpretación. Sigfried Giedion describe este papel del artista así:

El artista, de hecho, funciona mucho como un inventor o un descubridor científico: los tres buscan nuevas relaciones entre el hombre y su mundo ... Por un lado el artista creativo no quiere copiar su entorno pero, por el otro, tampoco hacernos ver a través de sus ojos. [El artista] es un especialista que nos muestra en su trabajo como en un espejo algo que no nos hemos dado cuenta por nosotros mismos: el estado de nuestras propias almas ... Esta es la razón por la cual todavía necesitamos artistas, por difícil que sea para ellos mantener su lugar en el mundo moderno ${ }^{8}$.

En otras palabras, el acto artístico (y arquitectónico) requiere 'interpretación'. A pesar de las definiciones del diccionario, la interpretación no es explicación. La explicación implica que se ha alcanzado el significado final, la razón o la naturaleza de lo que esta siendo explicado y por lo tanto que no queda nada más por entender. El concepto de explicación radica en la creencia de que, en última instancia, podemos comprender una realidad que se considera como objetiva. El acto de explicación destruye el acto de interpretación al hacer que todo sea factual, verificable y, por lo tanto, sin necesidad de mayores esfuerzos de cuestionamiento. La explicación vacía, resta, priva a los individuos de sus roles en la interpretación de la realidad. Es la creencia en la posibilidad misma de explicación lo que está detrás de la adopción de la reproducción, copia, imitación como mecanismos de producción artística o arquitectónica. Porque una vez que se ha logrado la verdad sobre algo, ¿hay algo más que hacer que no sea repetir esa verdad fielmente? La explicación genera conformidad, estados mentales no críticos y perezosos, y repetición sin sentido: todas ellas fuerzas terriblemente negativas contra el acto creativo. La explicación es un callejón sin salida epistemológico y se ha demostrado que es engañosa por las argumentaciones y hallazgos filosóficos y científicos del siglo 20 (por ejemplo, la Escuela de Frankfurt, el Experimentalismo de Dewey, el Postestructuralismo, la Física Cuántica y Relativista, etc.). 
La interpretación, por otro lado, genera hábitos creativos de pensamiento y acción, aunque (a veces) con implicaciones difíciles de manejar. Como el consenso no está garantizado, la interpretación crea la real posibilidad de disonancia y ambigüedad cognitiva. En el peor de los casos, la interpretación provoca nihilismo, subjetivismo y/o relativismo radical. En el mejor de los casos, la interpretación abre nuevos espacios consensuales de pensamiento, emoción y acción. Siempre, la interpretación requiere compromiso, diálogo, trabajo, energía. La interpretación desactiva la conformidad y genera diversidad, creatividad y evolución.

El artista y el arquitecto necesitan transgredir, subvertir, trascender la explicación. Deben interpretar.

\section{Ejercicio de Diseño}

Aplicamos este conjunto de ideas en un ejercicio de diseño en un taller de arquitectura de primer año que dirigimos en la Universidad de Utah hace varios años. Partimos eligiendo pinturas específicas que podrían considerarse revolucionarias a las percepciones de su tiempo. Estas obras de arte, junto con sus autores, también fueron seleccionadas en base a sus diferencias temporales y culturales, con la intención de que provocaran discernimientos distintos. Las pinturas elegidas fueron: Sueño de Inocencio III de Giotto (circa 1240); La Cena de Emaús por Caravaggio (1600); Las Meninas de Diego Velázquez (circa 1640); Guernica de Pablo Picasso (1937); Plata Sobre Negro, Blanco, Amarillo y Rojo de Jackson Pollock (1948); y Hombre y el Niño de Francis Bacon (1963).

Somos conscientes de que hay precedentes en el uso de obras de arte como catalizadores creativos en el taller de arquitectura. Sin embargo, hasta donde sabemos, la mayoría de estas aplicaciones han dependido en gran medida de la documentación y las críticas existentes. Alternativamente, estos estudios se han centrado en análisis geométricos o compositivos (es decir, sintácticos) y manipulaciones carentes de significado (es decir, semántica). Además tales aplicaciones han enfatizado el resultado final del esfuerzo interpretativo. En tales precedentes el proceso que obviamente existió ha sido reconocido tácitamente pero no revelado como procedimiento de diseño e interpretación.

Por el contrario, en nuestro esfuerzo educativo utilizamos el proceso de transformación como un acto de interpretación que es activo y progresivo ('lectura') basado en su propio desarrollo y en la pintura misma. Aquí el énfasis estuvo colocado en cómo la 'lectura' evoluciona en un continuum, cómo se leía cada etapa en el contexto de las otras, y finalmente cómo se comprendía toda la transformación a la vez. Por lo tanto, la interpretación inicial del tema o cuestiones abordadas por un cuadro se transformaba significativamente por el acto de avanzar la interpretación en un proceso de etapas. El conocimiento existente (por ejemplo, la interpretación de Foucault de "Las Meninas") tomó una importancia cada vez más periférica a medida que el impulso del salto interpretativo de los estudiantes los alejó de su punto de partida (Figura 1). En verdad, no estábamos interesados en dónde aterrizaban los estudiantes después de saltar, sino en su vuelo. 


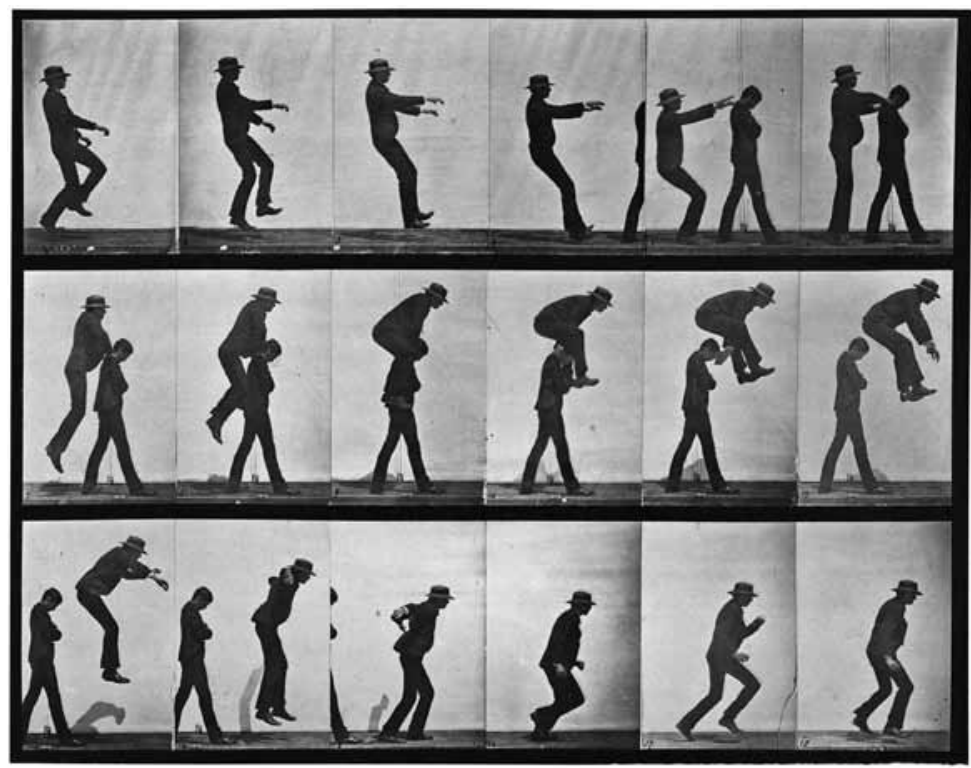

Figura 1. Locomoción Animal, Lámina 169 por Eadweard Muybridge (1887) ${ }^{8}$.

El ejercicio requería que los estudiantes pasaran de una pintura a la arquitectura utilizando el acto intencional de interpretación (es decir, la 'lectura') como su motor de diseño. Esta metodología claramente implicaba un salto metafórico que exigía involucrarse tanto en la abstracción como en el análisis, y tenía que manifestarse a través de medios apropiados y representaciones gráficas. De importancia esencial fue que el proceso de transición tenía que ser capturado en una serie de 'momentos congelados' (o 'transformaciones') que revelaran efectivamente las etapas críticas/de diseño de la investigación. El proceso podía documentarse en cualquier medio, desde la carbonilla hasta el collage y simulaciones electrónicas. La única limitación para los estudiantes era presentar su trabajo en un formato bidimensional para que (1) experimentaran la misma limitación planimétrica que los artistas; y (2) se vieran obligados a aclarar su visión arquitectónica, dada la mediación necesaria entre la arquitectura tridimensional y el campo de representación en dos dimensiones. Finalmente, organizamos a los estudiantes en grupos de 4 o 5 personas para garantizar una diversidad de interpretaciones ( $y$, por lo tanto, generar un verdadero debate de ideas), así como para tener la masa crítica suficiente para responder en calidad y cantidad a las demandas de productividad del taller.

Para comenzar nuestro trayecto pedagógico e invocar la fascinación y la curiosidad como combustible, les hicimos a los estudiantes algunas preguntas simples: ¿Qué tiene de arqui- 
tectónico una obra de arte en su contexto histórico general, o específicamente en la superficie condensada de una pintura? ¿Cuáles son los problemas arquitectónicos relevantes que surgen del cuadro? ¿Es su estructura espacial, técnica o compositiva? ¿O, más bien, es su contenido simbólico, temático y fenómenológico? ¿Cómo la 'lectura' de la obra informa la producción teórica y/o profesional de la arquitectura actual?

\section{Resultados}

A continuación, compartiremos brevemente los resultados generales del proyecto.

\section{Las Meninas}

Quizás la exploración más conocida de cómo el orden espacio-simbólico de una pintura desafía la percepción espacial del espectador es el análisis de Michel Foucault de "Las Meninas" de Diego Velázquez (Figura 2) ${ }^{10}$. El análisis verbal de esta pintura ha inspirado mucha intriga con respecto a su aplicación visual a la teoría arquitectónica. Debido a que esta pintura es posiblemente una de las primeras en ser utilizada en la búsqueda exhaustiva de entendimiento arquitectónico, sentimos que debía ser incluida en este ejercicio del taller.

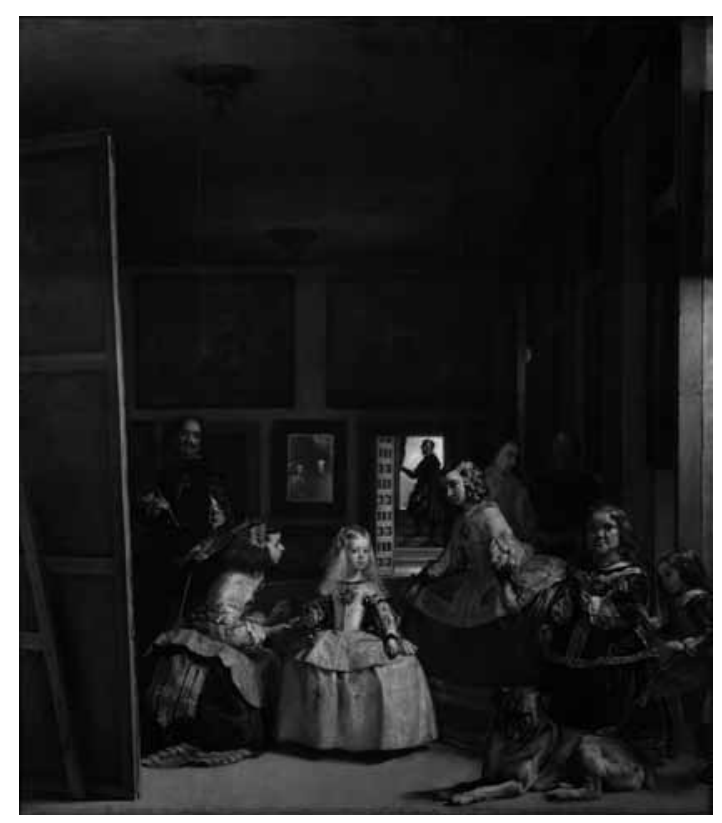

Figura 2. Las Meninas de Diego Velazquez (1656). 

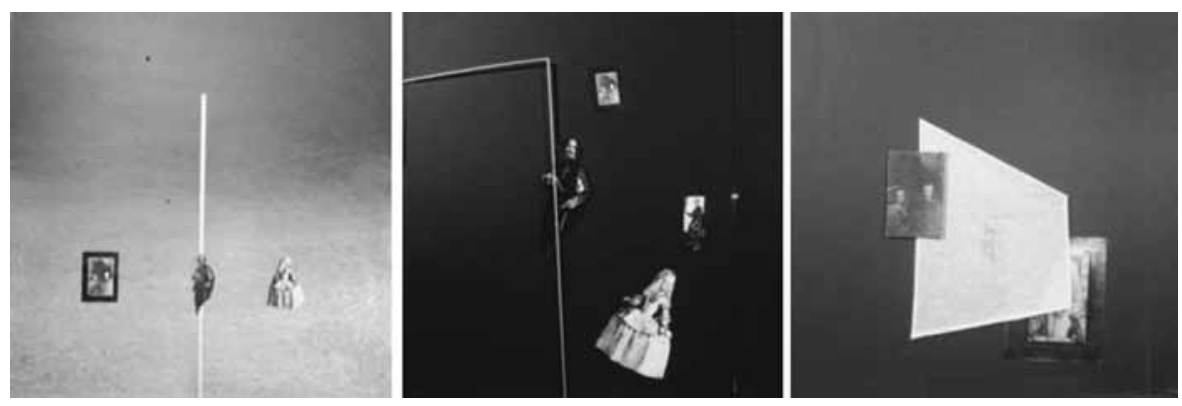

Figuras 3, 4 y 5.

En esta transformación de "Las Meninas" (Figuras 3, 4 y 5), los estudiantes exploraron el concepto de 'intermediación' refiriéndose específicamente a la relación de artista, sujeto y espectador (audiencia). El elemento clave de la transformación fue el plano del lienzo como espejo o lente. La etapa final presenta una comprensión de la pintura que desafía la lectura inicial de la obra, lo que sugiere que los primeros encuentros deben evaluarse cuidadosamente y quizás descartarse. Los estudiantes también se dieron cuenta de la naturaleza activa del hacer y el papel participativo de la audiencia.

Otro grupo de estudiantes que también trabajaba con "Las Meninas" investigó el eje que el lienzo niega (la profundidad) al usar primero la pantalla (es decir, el lienzo) para establecer un antes y un después, y luego guiar a la audiencia más allá del plano del lienzo hacia una sugerencia de espacio arquitectónico. La luz y la oscuridad fueron cuidadosamente estudiadas y reinterpretadas. Ambos grupos de estudiantes aprendieron a usar su curiosidad intuitiva para acceder a un mundo diferente. La arquitectura se presenta no como destino final sino como un conducto para continuar el viaje interpretativo.

\section{Hombre y el Niño}

Este cuadro proporcionó buenas oportunidades para reflexionar sobre la naturaleza de la representación arquitectónica (Figura 6). Aquí se prestó atención a (1) cómo se utilizaban los medios, la técnica y la composición para avanzar una agenda conceptual relacionada con el espacio-tiempo; (2) el acto de abstracción necesario para generar y construir el espacio pictórico; y así (3) cómo las representaciones arquitectónicas y artísticas son universos de discurso dentro de los cuales ciertos mensajes pueden ser transmitidos selectivamente (el medio es el mensaje) (Figura 6).

Los estudiantes tradujeron las formas de Bacon en una sintaxis arquitectónica utilizando convenciones ortográficas (plano, cortes y fachadas) para guiar y empujar su interpretación semántica (Figuras 7 y 8). La transformación pasa de un análisis objetivo tridimensional de la pintura (es decir, la representación como explicación) a una composición 


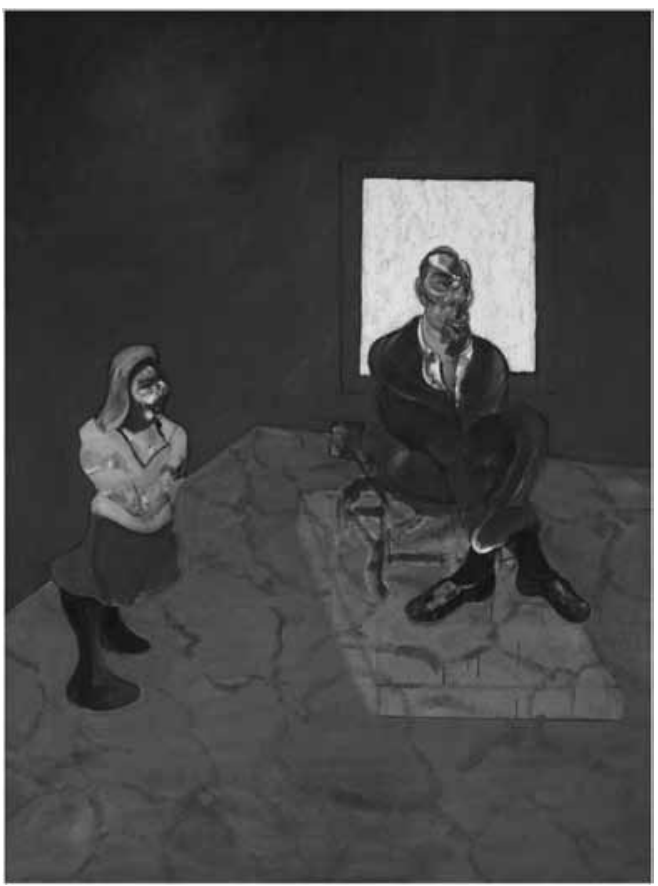

Figura 6. Hombre y el Niño de Francis Bacon (1963).
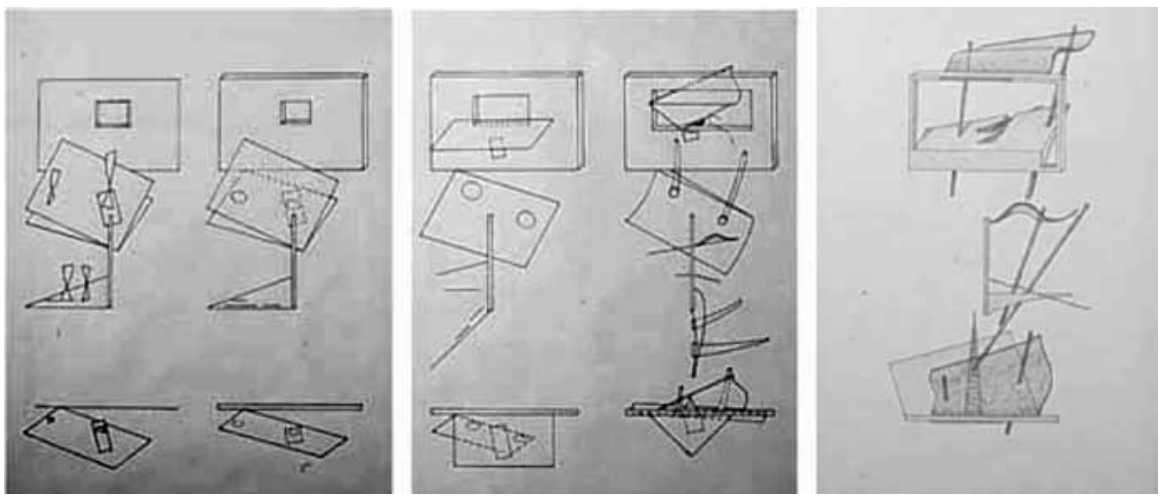

Figuras 7, 8 y 9. 
arquitectónica totalmente nueva (o sea, la representación como interpretación) (Figura 9). El catalizador de este proceso fue la decisión de los estudiantes de tomar posesión de la pintura al establecer sus propias reglas que se inspiraron o reaccionaron contra las implicaciones sociales de la agenda conceptual de Bacon.

\section{Sueño de Inocencio III}

Los estudiantes fueron sorprendidos por la calidad del collage, el uso de la semántica arquitectónica y el fuerte mensaje político detrás de la pintura de Giotto (Figura 10). La resolución del ejercicio se produjo cuando los diseñadores pasaron de una interpretación espacial-formal tradicional de la arquitectura a una de la arquitectura como política. Los estudiantes descubrieron que los principales impulsores de la arquitectura a menudo son fuerzas no arquitectónicas. Pero al igual que Giotto no solo estaba documentando la historia como se le pidió que hiciera, sino que la criticaba, los estudiantes se dieron cuenta de que los arquitectos también pueden desempeñar un papel ideológico importante si establecen un diálogo crítico con las fuerzas culturales del presente.

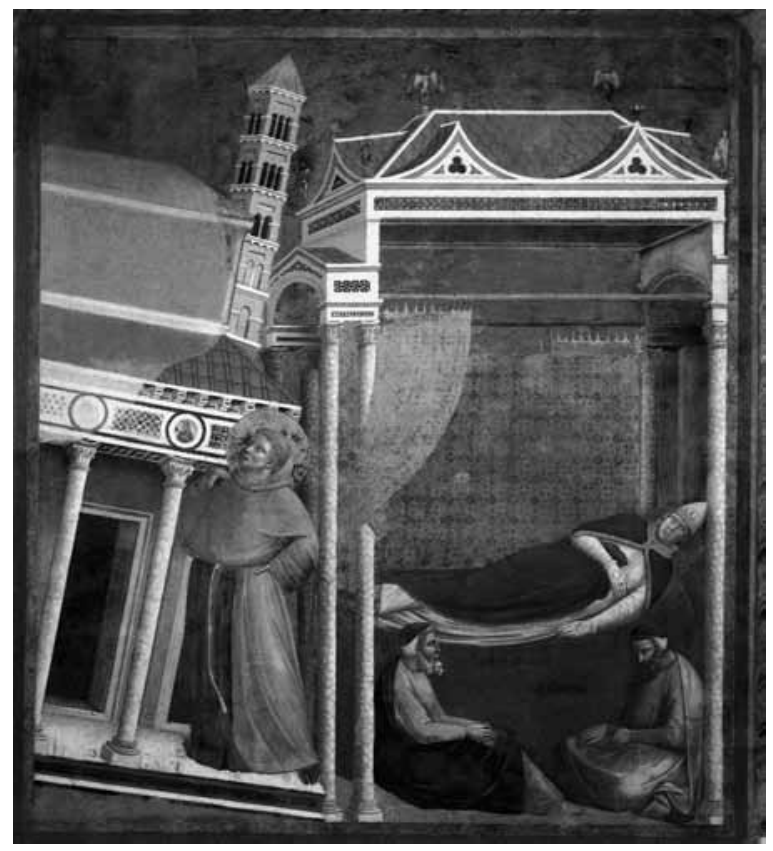

Figura 10. Sueño de Inocencio III de Giotto (1297-1299). 

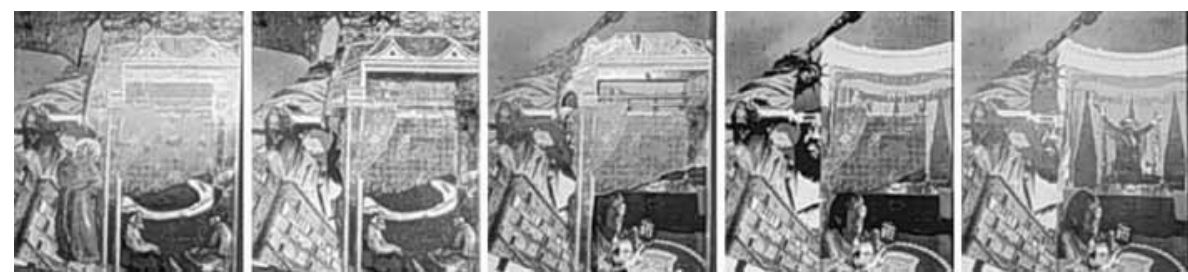

Figuras 11, 12, 13, 14 y 15.

La transformación de los estudiantes (Figuras 11 a 15) genera una serie de asociaciones paralelas entre el mundo sociopolítico de Giotto y el actual. Cada símbolo en la pintura encuentra su contraparte contemporánea. Alcanzar el consenso sobre la selección apropiada provocó acaloradas discusiones y logró que los principiantes de arquitectura despertaran a la realidad política del pensar y hacer constructivo, un tema que a menudo se pierde en los talleres de diseño, especialmente aquellos en el comienzo de la carrera.

\section{Plata sobre Negro, Blanco, Amarillo y Rojo}

La naturaleza aparentemente caótica de la pintura de Pollock (figura 16) puso a los estudiantes en la situación de tener que idear algún sistema de interpretación para darle sentido al trabajo. La clave para comprender la implicación del cuadro era entender la metodología de ejecución del artista (que se establece de hecho en el título de la obra). Esto significó explorar la naturaleza, la secuencia y los roles potenciales de las diferentes capas.

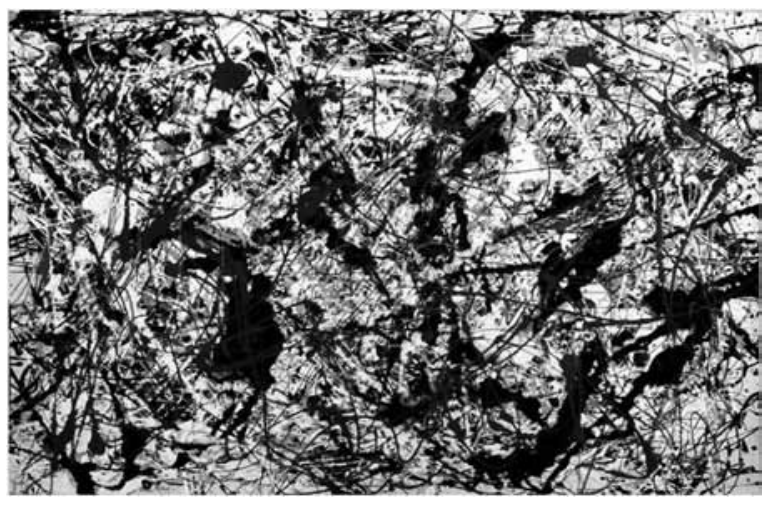

Figura 16. Plata sobre Negro, Blanco, Amarillo y Rojo de Jackson Pollock (1948). 

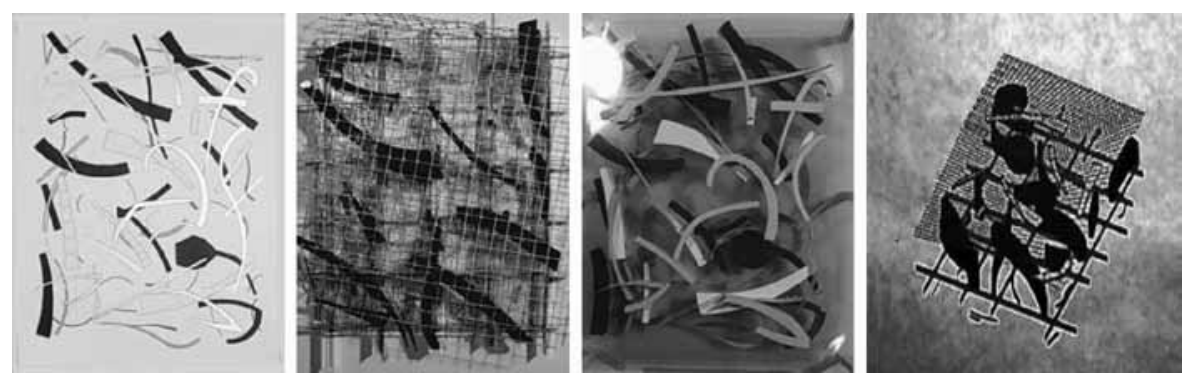

Figuras 17, 18, 19 y 20.

Los dos proyectos producidos en el taller incorporaron el evidente método de pintar de Pollock en su presentación. Sus diferentes resultados fueron una consecuencia directa de las elecciones de medios que hizo cada equipo: medios digitales versus medios análogos. Mientras que la producción electrónica se centró en el despliegue temporal de la realización pictórica (es decir, una simulación temporal del pintar de Pollock, una animación), la producción física consideró la presencia simultánea del resultado (o sea lo sincrónico). Mientras que el primero estaba interesado en las cualidades ocupables del espacio en capas (Figuras 17 a 20), el segundo prestó atención a las cualidades formales del todo pictórico. En otras palabras, el trabajo digital se ocupó de lo fenomenológico mientras que la interpretación física se ocupó de los aspectos conceptuales de la obra.

\section{Conclusiones}

El ejercicio cumplió exitosamente con las premisas pedagógicas citadas. Los estudiantes inicialmente dudaron de su capacidad de hacer observaciones apropiadas. Sin embargo rápidamente ganaron confianza y propusieron cuestionamientos que generaron un diálogo prolífico, contagioso y emocionante. El alto nivel de producción es un buen indicador de cuánto interés y compromiso despertó este proyecto en los estudiantes dado que fue de solamente una semana de duración.

Se pueden citar cuatro enfoques interpretativos:

1. sintáctico/conceptual (por ejemplo, Bacon, la interpretación gráfica de Pollock);

2. político/simbólico (por ejemplo, Giotto);

3. fenomenológico/empático (por ejemplo, Velázquez, Bacon, la interpretación digital de Pollock); y

4. metodológico (por ejemplo, Giotto, Pollock). 
Observamos una gran resonancia afectiva mientras los estudiantes trabajaban y discutían sobre las pinturas de Giotto y Bacon. El "Sueño de Inocencio III: despertó inesperadamente el juicio, la controversia y el rechazo entre los estudiantes a medida que surgían desacuerdos políticos serios, particularmente con el rol de las instituciones religiosas en el país y mundo de hoy. El tono emocional acalorado de los intercambios fue muy inusual en el momento en que se realizó este proyecto, que era muy diferente al clima político que vivimos hoy en 2019. El cuadro "Hombre y el Niño" generó un tipo diferente de sentimientos, en este caso asociado con la naturaleza espeluznante, misteriosa, sino negativamente sublime de la imagen. La decisión de los estudiantes de eliminar las asociaciones figurativas en su proceso de transformación fue su manera de alejarse de las emociones incómodas, también preocupantes, que originó la pintura de Bacon. Mientras que los dos cuadros anteriores indujeron reacciones emotivas hacia el tema de la pintura o la imagen en sí, los estudiantes aún podían mantener un punto de vista en tercera persona. "Las Meninas" abrió la posibilidad de una perspectiva en segunda persona que invitó a la empatía, ya que los estudiantes pudieron asumir la personalidad de los diversos personajes del cuadro algo facilitado por la naturaleza fotográfica de la obra de arte. Los estudiantes también se vieron obligados a alternar posiciones entre el pintor (en la imagen) y el público, obteniendo importantes discernimientos fenomenológicos sobre la relación interdependiente entre situación, realidad y punto de vista. El trabajo de Pollock creó una manera muy diferente de respuesta empática. En este caso, los estudiantes experimentaron la sensación corporal de arrojar óleo por el aire y sobre el lienzo y percibir cómo lo aparentemente fortuito seguía los movimientos 'lógicos' del cuerpo humano y la gravedad. Unos pocos se dieron cuenta de cómo tales actos capturaban la fugacidad existencial de la vida en ese mismo instante.

En otras palabras, a pesar del hecho de que el esfuerzo interpretativo se basaba en la percepción sensual y generalmente avanzaba a través del esfuerzo conceptual y analítico, este ejercicio de taller permitió a los estudiantes aceptar, y a veces centrarse, en el poder y la importancia de las emociones. Esto fue, por supuesto, intencional. La ciencia educativa establece claramente que el aprendizaje mejor o más profundo sucede cuando el cerebro, el cuerpo y el corazón se incorporan en un proceso que también incluye un componente social significativo, como es el caso de este ejercicio de diseño. La riqueza de interpretaciones demostró a los estudiantes que la visión arquitectónica se puede obtener de muchas maneras diferentes. Les enseñó una forma de acercarse a la arquitectura que no depende de la arquitectura. Como resultado, este proyecto también avanzó su alfabetización visual en significado y simbolismo. Igualmente importante, el ejercicio demostró a los estudiantes que las ideas se descubren y evolucionan a través de un proceso de estudio crítico y negociación social y no en un acto instantáneo de explicación concluyente. Con la confianza ganada en el acto de descubrimiento, la fluidez de la ideación (la creatividad) aumentó en los proyectos de taller que siguieron a este.

En este taller, el arte fue una fuerza de liberación que desactivó las preconcepciones que tanto debilitan a la creatividad y son tan presentes en los principiantes de arquitectura, y al mismo tiempo los instruyó en como transformarse en intérpretes comprometidos y no en imitadores de nuestro mundo. 


\title{
Notas
}

1. Publication Reference: McDonough, Peggy and Bermudez, Julio (1996). “Abstraction . .. Morphosis ... Inspiration, Art As A Source Of Architectural Insight In The Design Studio"; in Proceedings of the 13th. National Conference on the Beginning Design Student. Baton Rouge, LA: Lousiana State University, pp. 275-279.

2. Colin Rowe y Robert Slutzky, "Transparency: Literal \& Phenomenal," en The Mathematics of the Ideal Villa and Other Essays, ed. Colin Rowe (Cambridge, MA: The MIT Press, 1976), pp. 159-183.

3. Ibid., Rowe y Slutzky prestan especial atención a la idea de la transparencia fenomenológica, en el sentido de que las figuras, los volúmenes arquitectónicos, por ejemplo, son "capaces de interpenetrarse sin destrucción óptica entre sí" durante la experiencia arquitectónica. Esta idea tiene su origen en el artista Gyorgy Kepes.

4. Por ejemplo, Sigfried Giedion, Space, Time \& Architecture (Cambridge, MA: The Harvard University Press, 1941). Christian Norberg-Schulz, Meaning in Western Architecture (New York: Praeger Publishers, 1975).

5. Daniel Libeskind, The Edge of Order (New York, Crown Publishing Group, 2018). Juhani Pallasmaa, Encounters (Rakennustieto Publishing, 2008).

6. Twyla Tharp con Mark Reiter, The Creative Habit (New York, NY: Simon \& Schuster, 2003) pp. 4-7.

7. Garth Rockcastle y Mary A. Dixon-Hinson, “The Value of Type: A Debate," Midgärd Monograph: Type \& the (Im)Possibilities of Convention (Princeton, NJ: Princeton University Press, 1991) pp.8-9. Traducción al castellano por Julio Bermudez.

8. Giedion, Space, Time \& Architecture, p. 432. Traducción al castellano por Julio Bermudez. 9. Eadweard Muybridge, Animal Locomotion, Plate 169, circa 1920, procedente de la Biblioteca Pública de Boston, Estados Unidos (imagen en el dominio público).

10. Michel Foucault, The Order of Things: An Archaeology of the Human Sciences (New York, NY: Pantheon Books, 1970), pp. 3-16.

\begin{abstract}
Although much has been said about the direct relationship between art and architecture, little has been done regarding how historically significant works of art may be utilized as sources of inspiration for the architectural production of today. We may cite several historical and aesthetic analyses on such works of art and their particular zeitgeist, but how might such a work of art illuminate and extend today's architectural process, theories and ideas?In other words, (1) how does the past (in)(de)(re)form the present? and (2) how do another discipline's insights(in)(de)(re)form our discipline's process of ideation? Having these thoughts in mind, a pedagogy was developed tomake students realize that (1) architecture deals with issues and ideas that transcend the purely programmatic (i.e.'functional') and the stereotypically formal; (2) new architectural ideas may come from old and non-architectural sources; (3) ideas are discovered and evolve through a staged critical process, as opposed to a singular act of explanation ; and (4) the role of
\end{abstract}


the architect/designer is one of interpreter, as opposed to imitator, of one's surroundings, culture, tradition, etc.

Keywords: art and architecture - design pedagogy - project process.

Resumo: Embora tenha sido dito muito sobre a relação direta entre arte e arquitetura, pouco foi feito sobre como as obras de arte historicamente significativas podem ser usadas como fontes de inspiração para a produção arquitetônica atual. Podemos citar várias análises históricas e estéticas de tais obras de arte e seu espírito particular, mas como essa obra de arte poderia iluminar e estender o processo arquitetônico, as teorias e idéias atuais?

Em outras palavras, (1) como o passado (in) (de) (re) forma o presente? e (2) como as percepções de outra disciplina (in) (de) (re) formam o processo de ideação de nossa disciplina? Levando esses pensamentos em consideração, uma pedagogia foi desenvolvida para permitir que os alunos no início da carreira de arquitetura percebam que (1) a arquitetura aborda questões e idéias que transcendem o puramente programático (ou seja, 'funcional') e o estereotipicamente formal; (2) novas idéias arquitetônicas podem vir de fontes não arquitetônicas do passado; (3) idéias são descobertas e evoluídas através de um processo crítico em estágios, em oposição a um ato singular de explicação; e (4) o papel do arquiteto / designer é interpretar, ao invés de imitar, o ambiente, a cultura, a tradição etc.

Palavras chave: arte e arquitetura - pedagogia do design - processo de projeto.

[Las traducciones de los abstracts fueron supervisadas por el autor de cada artículo] 